\title{
The future, and what might have been
}

\author{
R. A. Briggs ${ }^{1}$ - G. A. Forbes ${ }^{2}$
}

Published online: 30 January 2018

(C) The Author(s) 2018. This article is an open access publication

\begin{abstract}
We show that five important elements of the 'nomological package'laws, counterfactuals, chances, dispositions, and counterfactuals-needn't be a problem for the Growing-Block view. We begin with the framework given in Briggs and Forbes (in The real truth about the unreal future. Oxford studies in metaphysics. Oxford University Press, Oxford, 2012), and, taking laws as primitive, we show that the Growing-Block view has the resources to provide an account of possibility, and a natural semantics for non-backtracking causal counterfactuals. We show how objective chances might ground a more fine-grained concept of feasibility, and furnished a places in the structure where causation and dispositions might fit. The Growing-Block view, thus understood, provides the resources to explain the close link between modality and tense, so that it predicts modal change as time passes. This account lets us capture not only what the future might hold for us, and also what might have been.
\end{abstract}

Keywords Growing-Block · Laws · Counterfactuals · Chance · Dispositions · Causation

Imagine finding yourself nostalgic; you recall a time in your life when the future still seemed wide open. You wonder 'what if I had done something else?'. The move from the open future to the possible past seems a very natural one, and it would be useful for the philosophy of time to explain the link between tense and modality. The kind of modality that interests us shows up in a variety of concepts:

G. A. Forbes

G.A.Forbes@kent.ac.uk

1 Department of Philosophy, Stanford University, Stanford, CA, USA

2 School of European Cultures and Languages, University of Kent, Canterbury, UK 
laws of nature, counterfactuals, dispositions, causation, chance, and of course, the concept of nomological possibility. We shall refer to the collection of categories that are modal (in the relevant sense) as the nomological package. ${ }^{1}$

One theory that offers to explain the link between tense and modality is the Growing-Block view, which appeals to metaphysical claims about time (in particular, the claims that only past and present things and events exist, and that the passage of time consists of new things coming into existence) to capture a modal intuition (that possibilities in the future are different from possibilities in the past). The future is open; the past is not. Any defender of the Growing-Block view should be most interested in 'what if', 'will' and 'would have'.

Our aim, then, is to show how the Growing-Block view gives us the resources to talk about certain elements of the nomological package-in particular, laws, counterfactuals, and chance. Section 1 briefly lays out the Growing-Block view, as developed in Briggs and Forbes (2012). We won't attempt a defense of the view here, but extended defenses can be found in Broad (1923), Tooley (1997), Button (2006), Correia and Rosenkranz (2013), and Forbes (2016). Section 2 explains how the view can be combined with a non-Humean account that fits central elements of the nomological package together.

\section{The Growing-Block view}

The Growing-Block view has two central commitments: first, that past and present events and things exist, while future events and things do not; and second, that the passage of time consists of new events and things coming into existence. Thus, it is essentially a dynamic view, on which the ontology of the world undergoes a fundamental change as time passes. We claim that on the Growing-Block view, what is possible undergoes a fundamental change as well. We will rely on the version of the view we developed in Briggs and Forbes (2012).

\subsection{A summary of the Briggs-Forbes view}

Although there are no future events or things, there are non-trivial truths about the future. These truths are made true by events and things in the past and present, plus the laws of nature (Briggs and Forbes 2012, 298). Truths about the future can be characterized using ersatz possible worlds called timelines, modelled on the worlds of Adams (1974).

While the actual world - the one that is continually growing as time passes-is a collection of concrete times arranged in a temporal order, ersatz timelines are collections of abstract times arranged in temporal order. These ersatz timelines are static and unchanging. As time passes, a series of different ersatz timelines come to represent the same growing concrete world, and then cease to fully represent it (though they still represent proper parts of it).

\footnotetext{
$\overline{1 \text { This list is representative, but may not be exhaustive; see Sect. 2.6. }}$
} 
Each possible concrete time can be represented by an ersatz time, a set of tensed atomic propositions in a suitably ideal language. (We can think of these propositions as consisting of $n$-place predicates applied to names.) We built a larger language $\mathcal{L}$, consisting of

- names $a, b, c, \ldots$,

- variables $x, y, z, \ldots$,

- $n$-place predicates (for arbitrary $n$ ) $R_{1}^{1}, R_{1}^{2}, \ldots R_{1}^{2}, R_{2}^{2}, \ldots R_{1}^{n}, R_{2}^{n}, \ldots, \ldots$

- the connectives $\wedge, \vee, \neg$, and $\supset$,

- tensed quantifiers $\forall$ and $\exists$,

- tenseless quantifiers $\Sigma$ and $\Pi$, and

- tense operators, including the past and future operators $P$ and $F$

Tensed propositions in $\mathcal{L}$ take their truth values relative to both a time and a timeline (What is true simpliciter at a timeline is what is true at the present- the last moment in the timeline). ${ }^{2}$

One ersatz timeline is actualized-i.e., accurately represents all and only the things in the concrete actual world. Other ersatz timelines are feasible-they not only accurately represent the concrete actual world, but also things and events that may come to exist in the future. Timelines can be parts of other timelines, and every feasible timeline has the actualized timeline as a part: the actualized timeline forms the initial segment of every feasible timeline. If $T$ is an initial segment of $T^{*}$, we say that $T^{*}$ is an extension of $T$.

Each timeline, when taken together with all of its feasible extensions, generates a tree-like model structure composed of partially overlapping timelines. Figure 1 depicts the actualized timeline, together with its feasible extensions, as are truncated branches of a tree which begin at the root, but need not extend all the way outward to a leaf. The actualized timeline runs from $e_{0}$ to $e_{1}$. One feasible timeline runs from $e_{0}$ to $e_{2}$ and stops; another runs from $e_{0}$ all the way to $e_{3} .^{3}$

We drew further distinctions among the timelines in the branching structure. Some are incomplete, and must continue growing, while others, after their final big crunch or whathaveyou, are incomplete and can grow no more. Timelines that could end where they are, but could be continued, are semi-complete.

Some timelines in the branching structure are 'histories'-either complete timelines, or semi-complete timelines viewed 'as histories'. Intuitively speaking, histories are maximally specific ways the original timeline could turn out. Histories are classical-at every time in every history, every proposition is either true or false. Conjunction, negation, and the universal and existential quantifiers take their usual classical semantic values, and tense are given Priorian modal definitions $P$ and $F$ (so

\footnotetext{
2 The ersatz time that we have been treating as present could in fact be the limiting case of the past. That is, it could be that what is true at the present moment is whatever is true at some time span that is not succeeded by any other time span, and true at all shorter time spans that are not succeeded by any other time span. (On this way of speaking, you are now reading a paper about time because you've been reading a paper about time for the last minute, and the last $30 \mathrm{~s}$, and the last $15 \mathrm{~s} \ldots$ and so on.) Treating the present as the limit of the past should work for most practical purposes.

${ }^{3}$ We use $e$ for ersatz times, to emphasize their difference from concrete times.
} 


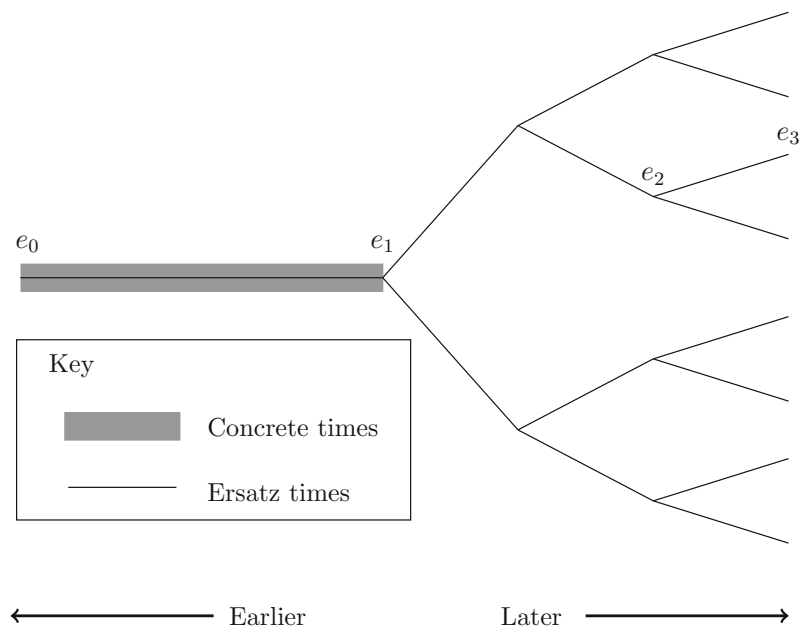

Fig. 1 The actual timeline and its feasible timelines

that, for example, $P \phi$ is true at a time in a history whenever $\phi$ is true at at least one earlier time in that history, and $F \phi$ is true at a time in a history whenever $\phi$ is true at at least one later time).

We propose three possible ways of extending this classical definition to obtain a definition of truth at a time in an arbitrary timeline, but for simplicity's sake, we will adopt the supervaluational semantics, modeled after Stalnaker and Thomason (1970), here. A sentence is true at an ersatz time $e$ in a timeline $T$ if it is true at $e$ in every history that is a feasible extension of $T$, false at $e$ in $T$ if it is false at $e$ in every history that is a feasible extension of $T$, and indeterminate otherwise. ${ }^{4}$

This should suffice as enough background for the work we intend to do. In the next subsection, we will outline the elements of the nomological package that we think the Growing-Block theory is best poised to explain, and say how a GrowingBlock theorist ought to model them.

\section{A non-Humean account of the nomological package}

The nomological package is a collection of intertwined modal concepts. In our (2017) we argued that a Growing-Block theory of future truth is incompatible with Humeanism - the claim that facts about elements of the nomological package (such as laws, nomological possibility, counterfactuals, and chances) supervene on nonnomological facts. So a Growing-Block theorist must appeal to fundamental modality. What can Growing-Block theorists say about this fundamental modality to render it less mysterious?

\footnotetext{
${ }^{4}$ In this paper, we will focus on models in which there are a finite number of times, and we will ignore the 'inevitability' operators from our earlier account, which create special complications.
} 
Quite a lot, we think. The remainder of this section lays out a model of modality for Growing-Block theorists. We understand laws as true sentences in a timeless language, whose truth is grounded by parts of reality called lawmakers (Sect. 2.1), use the laws to define the modality of feasibility (Sect. 2.2), and analyze (nonbacktracking causal) counterfactuals in terms of feasibility (Sect. 2.3). We explain how this basic picture can be modified to leave room for chances (Sect. 2.4), as well as causation and dispositions.

Figure 2 depicts grounding relations among the concepts we discuss; an arrow from $A$ to $B$ means that $A$ grounds $B$. For causation and dispositions, we suggest several alternative options, drawn in grey, and in parentheses: they might function as lawmakers, or be phenomena susceptible to analysis in counterfactual terms. Or causation might play a completely different role, either grounding or being grounded by feasibility.

We don't purport to offer a complete theory of any one element of the nomological package, although we will have novel things to say about laws, counterfactuals, and chance. Rather, our chief aim is to show how these separate pieces fit together into a harmonious picture, unified by the Growing-Block view.

\subsection{Laws}

To fully capture the concept of laws, we will need to make one modification to our earlier account. There are truths about the laws of nature, and these truths are arguably logically and metaphysically contingent. We will therefore assume that each ersatz timeline contains, in addition to an ordered sequence of times, a collection of laws, which we represent as sentences.

Fig. 2 Relations of grounding among elements of the nomological package

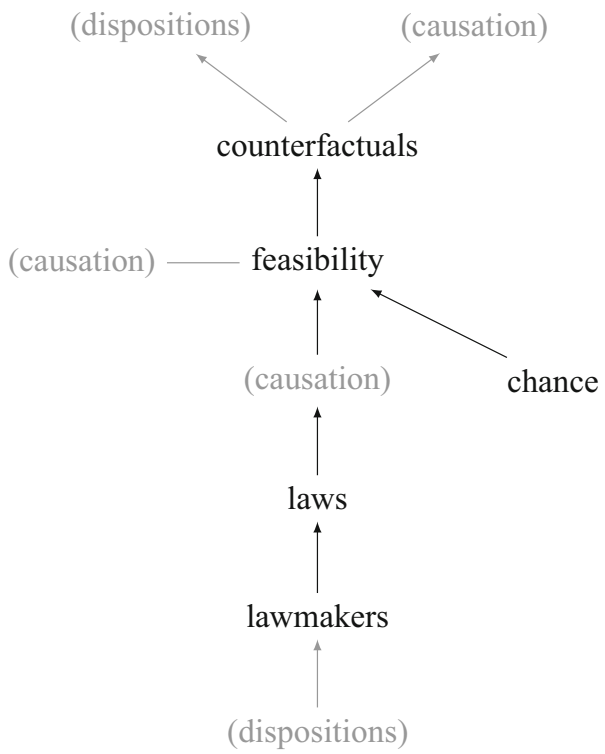


In our (2012) we defined our valuation function for tensed propositions, but the laws are timeless. Therefore, we will need to formulate a theory of tenseless propositions, which take their truth values at worlds alone. While ersatz times were sets of tensed sentences (which, intuitively, describe the world from the perspective of a particular time), our ersatz laws are tenseless sentences (which, intuitively, describe all the times from an outside, God's-eye perspective.)

We can modify the language $\mathcal{L}$ to create a language $\mathcal{L}^{*}$ of laws. The vocabulary of $\mathcal{L}^{*}$ consists of the vocabulary of $\mathcal{L}$ plus

- the symbol at,

- tenseless quantifiers $\Pi e$ and $\Sigma e$ that range over times, and

- the two-place predicates $E$ (earlier) and $L$ (later)

We can recursively define a well-formed formula in $\mathcal{L}^{*}$ as follows.

- if $\phi$ is a wff of $\mathcal{L}$ not containing any names, then $\phi$ ate is a wff of $\mathcal{L}^{*} .5$

- if $\phi$ and $\psi$ are wffs of $\mathcal{L}^{*}$, then $\phi \wedge \psi, \phi \vee \psi$, and $\neg \phi$ are wffs of $\mathcal{L}^{*}$

- if $\phi$ is a wff of $\mathcal{L}^{*}$, then $\Pi x \phi$ and $\Sigma x \phi$ are wffs of $\mathcal{L}^{*}$.

- if $\phi$ is a wff of $\mathcal{L}^{*}$, then $\Pi e \phi$ and $\Sigma e \phi$ are wffs of $\mathcal{L}^{*}$.

- if $e_{1}$ and $e_{2}$ are variables for times, then $E e_{1} e_{2}$ and $L e_{1} e_{2}$ are wffs.

So each timeline includes a collection of timeless laws, alongside its sequence of ersatz times. If the timeline is to count as possible, the laws must be well-behaved. They must be closed under logical consequence (though they need not be complete, since not every matter is settled by the laws). They must also play nicely with the sequence of ersatz times: they must not be false of the block as that sequence represents it. It's slightly tricky to get the right meaning for 'false of', as the following example illustrates.

Consider a timeline $T$ in which there are three ersatz times, $a_{1}, a_{2}$, and $a_{3}$. Suppose $T$ 's laws include the following sentence.

(LT) $\Pi e_{1} \Sigma e_{2}\left(L e_{1} e_{2}\right)$

'For all every time $e_{1}$, there is a time $e_{2}$ which is later than $e_{1}$.'

In the schematic example, we do not want to say the sequence of times represents a state of affairs that is inconsistent with (LT). The block as $T$ represents it is consistent-while $a_{3}$ isn't yet succeeded by any time, the block will grow to ensure that it is. The present is going to be succeeded by a later time, even if that later time hasn't yet come into existence.

We can define what it is for a law to be false of a sequence of ersatz times using a two-stage procedure. First, we can give a standard Tarskian definition for 'true of'. We can talk (a little loosely) of assigning names and ersatz times (rather than concrete individuals and times) to variables in open sentences of $\mathcal{L}^{*}$. We can then

\footnotetext{
5 We require that the laws be name-free on the grounds that laws are general, and do not make references to particular individuals. 'No olive tree produces apples' is a candidate law (or at least, it's not barred from the status of law due to its form). 'No tree in Olivia's orchard produces apples' is not a candidate law, because it mentions Olivia by name.
} 
say that $\phi a t e$ is true relative to an assignment of variables at a sequence of times iff replacing the variables in $\phi$ with the appropriate names yields a sentence that is true at the time assigned to $e$ in $T$. (The truth values of more complex sentences are given recursively.) In our example, (LT) is not true of our sequence of times, since if we assign $a_{3}$ to the variable $e_{1}$, there is no way of assigning a time to the variable $e_{2}$ that will make the open sentence $L e_{1} e_{2}$ come out true in $T$.

Next, we can say that a sentence of $\mathcal{L}^{*}$ is false of a sequence of times if and only if it is not true of any extension of that sequence of times-i.e., just in case it will never become true. In or example, (LT) is not false of our sequence of times. A law is consistent with a sequence of ersatz times so long as it is not false of the sequence of ersatz times.

The apparatus developed so far lets us define the concepts of completeness, semicompleteness, and incompleteness we glossed in Sect. 1. We can say that a timeline is complete just in case its laws are true of it, but not true of any other extension of it; semi-complete just in case its laws are true of it and some of its other extensions; and incomplete if its laws are true of some of its extensions but not true of it.

So far, our discussion has been at the level of ersatz timelines-representations of ways the world might be. But ersatz timelines represent the concrete world, and can represent it either truly or falsely. We have an intuitive grasp on the truthmakers for tensed sentences: they are concrete things located at times, such as tables and chairs, cats and donkeys, cabbages and kings, that a tensed sentence can represent accurately or inaccurately. But what are the lawmakers - the features of the world that the laws can represent accurately or inaccurately?

We think the Growing-Block theorist has a variety of appealing options. The lawmakers might be dispositions (see Sect. 2.5 below), Aristotelian natures (see Cartwright 1999, 77-103), or capacities of individuals. They might be primitive facts picked out by structural equations (see Pearl 2009). Or they might not be nameable at all, except by pointing to the laws, and saying that lawmakers are whatever parts of reality make the laws true. In our (2017) we argued that for Growing-Block theorists, the lawmakers cannot be Humean entities. But that leaves plenty of space open for a positive characterization. What we have said here places very few constraints on the nature of lawmakers. But whatever the lawmakers are, they must constrain how the block grows.

\subsubsection{Objection: are we fundamentalists?}

We claim that our view of laws is admirably tolerant of the diverse opinions held by metaphysicians: all sorts of entities are qualified to be lawmakers, provided that they are strong enough to constrain the future. But objectors from some quarters might complain that our theory is too narrow. Why must laws constrain the future, instead of merely guiding it? These objectors side with (Cartwright 1999, 4), who claims that

1. our best scientific theories are true only in limited domains,

2. scientific laws hold only ceteris paribus, and 
3. laws lack the special epistemic status that some philosophers of science attribute to them: our knowledge of laws is less secure than our knowledge of the natures of things.

Cartwright's third point need not detain us; our Growing-Block theory concerns the metaphysics of laws, and says nothing about their epistemology. But her first and second points constitute a serious challenge. We have assumed that the laws of a timeline are true of the entire timeline (thus directly contradicting Cartwright's first claim), and that they are (nomologically) necessarily true (thus directly contradicting her second claim).

One possibility, of course, is that Cartwright's claims 1 and 2 are wrong. Her critics have marshaled a variety of arguments against 1 and 2. Mumford (2000) and Chakravartty (2003) point out that 1 and 2 are underdetermined by the scientific evidence, and that a priori considerations weigh against them. Teller (2002) questions two of the tacit premises in Cartwright's argument for claim 1-that scientific explanation proceeds from a relatively small stock of interpretive models, and that each of these models is naturally limited in its domain - adducing that these premises are at odds with Cartwright's evidence. Winsberg and Frisch (2000) argue that, contrary to claim 2, Cartwright's ceteris paribus laws can be converted into exceptionless laws with provisos as part of their content.

Even if 1 and 2 are true, however, there is no need to abandon the Growing-Block account. Cartwright does not argue for wholesale anti-realism about the nomological package. In fact, as she notes, scientific realism provides a good explanation for the success of our scientific theories. Rather, she claims that laws, as formulated in the language of current science and its most deserving successors, are neither unconditional (true necessarily, and not just ceteris paribus) nor unrestricted (applicable in all domains).

This leaves it open that there is some logically ideal language in which unconditional, unrestricted laws can be formulated. (Cartwright doubts that such laws would be simple or explanatory, but nothing we say requires the laws to be either.) Indeed, one of Cartwright's reasons for thinking that the laws are not true is that the language in which they are couched has imprecise application conditions which are impossible to codify.

Were there a notional language whose application conditions were precise, then there would be no obstacle to our formulating laws in that language. Granted, no living person speaks such a language, but as modal ersatzists, we are already committed to its existence. We think there are facts of the matter about which possibilities there are. For ersatzists, such facts can only obtain if there is some language in which complete descriptions of the world can be formulated, and something about the language that fixes which of those descriptions are consistent. While the language of our timelines is most likely not a language spoken by contemporary scientists, it must exist in some sense if the ersatzist view of modality is true. 


\subsection{Feasibility}

Philosophers are often interested in the restricted modal operators feasibility and nomological possibility. Growing-Block theorists can adopt a standard account of restricted modality: the $R$ possibilities (where $R$ can be filled in with 'nomological', 'feasible', or something else) are just the logical possibilities where some additional proposition is true. We can then characterize feasibility in terms of laws: nomologically possible timelines are those where the actual laws of nature hold.

But there is a crucial ambiguity here: do the actual laws hold in every nomologically possible world where they are true, or only in those worlds that have the status of laws? Humeans typically pick the first answer-the nomological possibilities are those in which the actual laws are true-because they are committed to the nomological possibility of undermining futures: sequences of events permitted by the actual laws that would fix different laws if they occurred. We are not bound by the strictures of Humeanism, so will say that the nomological possibilities are only those timelines in which the actual laws are laws. (We could, alternatively, make the two interpretations coincide by enriching the language of the laws to include a nomological necessity operator $\square$, and stipulating that $\square \phi$ is a law if and only if $\phi$ is a law.)

Feasible timelines are those that share both their history and their laws with the actual timeline. Feasibility is a particularly interesting modality because, on our version of the Growing-Block view, the laws and the past are fixed, while the future is open. So there is an important sense in which the feasible timelines are really possible - they might become actual as the block grows. Feasible timelines represent not merely counterparts of our actual world, but genuine possibilities for us.

\subsection{Counterfactuals}

Once we have a concept of feasibility, we can use it to analyze counterfactuals-or at least a certain subset of them. English-language counterfactuals run the gamut from 'If Emmy Noether and Sophie Germain were compatriots, then Noether would be French' to 'If I had jumped out my office window, I would have to have installed a net earlier to make sure that I would survive' to 'If there were only a finite number of primes, then it would be easier to sort marbles into groups of uniform size' to 'If you were to drop salt in water, then it would dissolve'. Not all of these counterfactuals are relevant to the nomological package.

Here, we are chiefly interested in non-backtracking counterfactuals whose antecedents are about events at a particular time. The Noether/Germain counterfactual in our list of examples is not of central interest, because its antecedent is not about the events at any particular time, but is instead about a global feature of the world; similarly for the prime numbers/marbles counterfactual. The window counterfactual is also not of central interest, because on its charitable reading, it backtracks, or ascribes a kind of non-causal dependence of the past on the future. We are most interested in counterfactuals like the salt/water example, which are laden with a type of causal nomological modality.

Following an idea pioneered by Stalnaker (1968) and Stalnaker and Thomason (1970), we will adopt a selection semantics for counterfactuals. The selection 
semantics is a possible worlds semantics in two senses: counterfactuals are classified as true or false at possible worlds, and their truth conditions are characterised in terms of possible worlds. Since our timelines resemble traditional possible worlds in some but not all respects, we will use 'world' for the entities in the traditional picture, and 'timeline' for the entities we ourselves are committed to. Throughout this section we will use the term 'actual' to designate the world or timeline at which the counterfactual is evaluated. (Since we have no reason to rigidly designate the timeline that authors and readers alike inhabit, the gain in ease of exposition outweighs the risk of confusion.)

The basic idea is this: the selection function maps each world $w$ and antecedent $A$ to a set of ' $A$ worlds' - i.e., worlds where $A$ is true. (To see how the world would have been if $A$ were true, we make the smallest possible change to the actual world that will ensure $A$ 's truth, and then consider all that this change entails.) The counterfactual $A \square \longrightarrow C$ ('if $A$ had been the case, then $C$ would have been the case') is true at $w$ if $C$ is true at all the selected $A$ worlds, and false at $w$ if $C$ is false at all the selected $A$ worlds. It's controversial what happens when the selected worlds disagree about $C$ 's truth value. Lewis (1973) claims that in this case, the entire counterfactual is false at the actual world, while Stalnaker (1981) says that it is neither true nor false. We adopt a version of Stalnaker's view: the selection function maps each timeline $T$ and antecedent $A$ to a set of timelines, and $A \square \longrightarrow C$ is true at a timeline $T$ if $C$ is true at all the selected timelines, false at $T$ if $C$ is false at all the selected timelines, and indeterminate otherwise.

So far, what we have said applies to all counterfactuals. But how shall we choose the selected timelines? Here, we shift our focus to the specific case of nonbacktracking counterfactuals whose antecedents are about events at a particular time. Examples might include:

- If Mary Anning had been killed by the lightning that struck her in 1800, Plesiosaurs would have remained unknown in England in 1821.

- If Empress Jia Nanfeng had kept Crown Prince Sima Yu under house arrest instead of having him assassinated, then she would have maintained her position of power within the Jin Dynasty for several more years.

- If you had kept the oven closed, the soufflé would not have fallen.

In each of these non-backtracking counterfactuals, the antecedent concerns (but need not mention explicitly) a specific time: 1800 in the first case; 300 AD (the year of Sima Yu's death) in the second; and the time of the soufflé's cooking in the third.

The Growing-Block theory suggests a 'branching time' account of such counterfactuals, of the sort developed by Thomason and Gupta (1981), defended by Leitgeb (2011a, b) and Loewer (2007), and discussed but ultimately rejected by Lewis (1979). ${ }^{6}$

\footnotetext{
6 This theory could be embedded in a more general theory of counterfactuals. There are counterfactuals whose antecedents are not about any particular time, but about a proposition that could be made true by a variety of events at different times, such as:
}

- If Maryam Mirzakhani or Tupac Shakur had lived longer, then the world would contain more beautiful artifacts. 
In metaphoric terms, our preferred interpretation of the selection function treats the Growing-Block like a stick of celery, with the earlier times nearer the base, and the later times towards the tip. Imagine the tip has gone bad: the antecedent of the counterfactual has turned out false. In order to get an idea of what the celery would look like healthy (with the antecedent true), you might cut off the soggy bits towards the tip, saving as much of the healthy celery as you can, and then imagine how the celery would regrow healthily.

To a first formal approximation, the procedure for evaluating a counterfactual $A \square \longrightarrow C$ at a timeline $T$, where $A$ concerns a particular time span, can be broken down into four steps.

1. Find a time $e_{A}$, located shortly before the beginning of the span.

2. Remove all the parts of the timeline after $e_{A}$ - thus generating a pruned timeline that represents the concrete block the way it was before it grew large enough furnish a falsemaker (or truthmaker) for the antecedent.

3. Consider all the feasible extensions of this shorter timeline-all the ways the concrete block might have grown-in which $A$ is true. The timelines generated by this method are our selected timelines.

4. If $C$ is true in all of the resulting timelines, then $A \square \longrightarrow C$ is true in the original timeline; if $C$ is false in all of the resulting timelines, then $A \square \longrightarrow C$ is false in the original timeline; otherwise, $A \square \longrightarrow C$ is neither true nor false.

The above procedure is an approximation, because it is important that the set of selected timelines be non-empty. What happens where there are no feasible timelines that coincide with the actual timeline up to $e_{A}$ in which $A$ is true? In this case, defender of branching-time counterfactuals faces a tricky choice. She can

Footnote 6 continued

This counterfactual is intimately connected to two others, whose antecedents are about events at particular times.

- If Maryam Mirzakhani had lived longer, then the world would contain more beautiful artifiacts.

- If Tupac Shakur had lived longer, then the world would contain more beautiful artifacts.

More generally, where $E$ is a set of events that could have occurred at particular times to make $A$ true, we can ask about the relationship between the counterfactual $A \square \rightarrow C$ and the counterfactuals of the form ' $e$ occurs $\square \rightarrow C$ ' for each $e \in E$. Following Briggs (2012) and Schulz (2011), we could say that $A \square \longrightarrow C$ is true just in case all of these counterfactuals are true. (This choice turns out to be incompatible with orthodox views about the logic of counterfactuals.) Or following ?, we could say that $A \square \longrightarrow C$ is true just in case the counterfactuals corresponding to the closest, likeliest, or most plausible events in $E$ are true. (This choice turns out to be compatible with orthodox views about the logic of counterfactuals.)

There are also counterfactuals whose antecedents are about propositions that are not made true at any time such as:

- If Gauss's Law were no longer a law of nature, then Coulomb's Law would no longer be a law of nature.

Our Growing-Block theory has less to say about these counterfactuals. In general, we subscribe to the view that selected timelines are those created by minimal changes to the actual timeline. A fully general theory would spell out the concept of 'minimal change' even when the change required had nothing in particular to do with the nomological package, but that is beyond our purview here. 
1. let the selected timelines differ from the pruned timeline in their history-i.e., the times before $e_{A}$,

2. let the selected timelines differ from the pruned timeline in their laws,

3. let the set of selected timelines be empty, or

4. let the selected timelines be impossible.

All four options have unappealing consequences.

Option 1 allows events in the distant past to depend counterfactually on events in future. Suppose it is not feasible for Leah to get a job teaching Mandarin: she does not speak Mandarin, and the few teaching jobs in her geographic area will go to better qualified candidates. And suppose that, when fed the actual timeline and the antecedent 'Leah gets a job teaching Mandarin', the selection function outputs worlds that have the same laws as the actual world, but differ from it in the distant past. Now consider an exhaustive state description $S$ of the world 510 million years ago, in the Cambrian era. In the actual world, $S$ is true, but in the selected worlds where Leah gets a job teaching Mandarin, $S$ is false. So the truth of $S$-a proposition that describes the world at the time of the trilobites-is counterfactually dependent on Leah's employment now. While Loewer (2007) attempts to soften the absurdity, this still seems like a serious bullet to bite.

Option 2 allows the laws of nature to depend counterfactually on occurrent history. Let $L$ be any true proposition that entails the complete system of laws in the actual timeline. Then, if Leah had gotten a job teaching Mandarin, $L$ would have been false. While Lewis (1979) attempts to soften the absurdity of this option, it too seems like a serious bullet to bite.

Option 3 makes all counterfactuals with infeasible antecedents come out trivially true. On this proposal, if Leah were to get a job teaching Mandarin, the sky would collapse, pigs would fly, and two plus two would equal five. We think this third option is not just costly, but prohibitively costly. Some claim that counterfactuals with metaphysically or logically impossible antecedents are trivially true (Williamson 2007; Lewis 1973); even this has its costs (for detailed discussion, see Nolan 1997; Brogaard and Salerno 2013; Krakauer 2012). To claim that counterfactuals with infeasible antecedents are trivially true is to compound the costs, since the infeasible propositions include all the impossible propositions, and plenty more. Option 3 is the worst of the four options, since it forces us to affirm all the unappealing counterfactuals affirmed by the other options, and more.

Option 4 lets the Growing-Block theorist affirm that even if Leah had gotten a job teaching Mandarin, the laws and the distant past would have remained the same. However, option 4 seems to force the conclusion that if Leah had gotten a job teaching Mandarin, something impossible would have happened. (The laws and the past would together have ruled out her getting the job, yet she would have got it anyway.)

All of options 1-4 land the branching time semantics in an awkward positionand indeed, they land a broader class of semantics in an awkward position. Any selection semantics for conditionals, where "if $A$, then $B$ " as true whenever $B$ is true at all selected worlds where $A$ is true, will have trouble in cases where $A$ is 
incompatible with history and the laws of nature. Our theory covers the central cases where $A$ is compatible with history and the laws of nature, and leaves 1,2 , and 4 open as options for the tricky cases.

\subsubsection{Indicatives}

Adams (1970) distinguished between indicative conditionals, like

(IND) If Stephanie Kwolek did not invent Kevlar, then someone else did. and subjunctive conditionals like

(SUB) If Stephanie Kwolek had not invented Kevlar, then someone else would have.

which now go by the name of 'counterfactual'. While our account is chiefly aimed at explaining counterfactuals, it explains features of indicatives too.

It is often remarked that in the future tense, there seems to be no distinction between indicatives and counterfactuals. Our branching-time theory goes a long way toward explaining this observation. Where $A$ is a proposition about the future, the second 'pruning' step of our procedure returns the actual timeline, whose feasible extensions are then selected. So when $A$ is a feasible proposition about the future, $A \square \longrightarrow C$ turns out to have the same truth value as $A \supset C$, on the supervaluationist semantics we defended in (2012).

The relationship between indicatives and counterfactuals breaks down when the antecedent $A$ is an infeasible proposition about the future. In this case, our proposed semantics for counterfactuals requires that (at least where $A$ is possible) the selection function assign a non-trivial truth value to $A \square \longrightarrow C$, while the semantics for indicatives in our (2012) entails that the indicative is trivially true. We think divergence is plausible in such cases - see the examples in Dorothy (2004) and Morton (2004)_but philosophers who disagree could tweak the semantics for indicatives so that $A \square \longrightarrow C$ is true at $T$ iff $C$ is true at every member of a set of $A$ timelines picked out by the selection function at $T$ - the set of feasible $A$ timelines if there are some, and the set of least-divergent $A$ timelines otherwise.

So far, Sect. 2 has given an account of (some) counterfactuals and their relationship to indicatives. We turn now to two objections which claim that our proposal gives the wrong answers about the counterfactuals within its scope.

\subsubsection{Objection: inflexibility}

Lewis (1979) raises an objection to branching-time accounts of counterfactuals like ours: they build temporal asymmetry into the analysis of counterfactuals by fiat. But this is 'too inflexible' since we can imagine and believe in various kinds of time travel to the past, precognition, and reverse causation.

We don't think it's too inflexible. The Growing-Block theorist is already committed to a deep, metaphysical asymmetry between the past, which is fixed and unchanging, and the future, which is open. This already rules out time travel to the past, precognition, and reverse causation. Therefore, the Growing-Block theorist need not balk at the metaphysical commitments of our proposal. 


\subsubsection{Objection: Morgenbesser cases}

Another objection to our proposal is that it gives the wrong answers in so-called Morgenbesser Cases (after an example by Sidney Morgenbesser, reported in Slote 1978). Suppose I bet at $e_{1}$ that a particular coin will land tails on the next toss. At $e_{2}$, a few seconds later, the coin lands heads. My bet has no influence on the mechanics of the coin toss; the bet and the coin toss take place in two different rooms, causally isolated from each other. Intuitively, it seems true that

BET If only I had bet heads instead of tails, I would have won!

But according to our proposal, BET is not true. The outcome of the coin toss is fixed after my decision to bet. So among timelines

(a) that are feasible extensions of the segment of the actual timeline that ends shortly before my bet, in which

(b) I bet heads,

there will be some timelines in which the coin lands tails.

Our Growing-Block semantics can be adjusted to accommodate the Morgenbesser intuition, and at the end of this section, we explain how. However, we don't wish to make this adjustment. The Growing-Block theorist should claim that the Morgenbesser intuition is mistaken, rather than trying to accommodate it.

Notice that when the coin toss is causally determined, but chaotic and so hard to predict, our account says that BET is true. Any way of modifying the bet that minimizes violations of the actual laws will leave the determining causes of the coin toss intact (After all, these determining causes are distant and causally isolated from the bet).

Only in indeterministic case does our account give the counterintuitive result that BET is false. However, it is not clear that intuition gets things right in this case. ${ }^{7}$ Consider the timeline whose present is just before the bet is placed, when there is no fact about how the coin will land. In that timeline, the disjunction

BET $^{\vee}$ Either I will not bet heads, or I will win!

is neither true nor false, but has indeterminate truth-value. Furthermore, the indicative conditional

BET $^{\supset}$ If I bet heads, then I will win!

has indeterminate truth value. But if BET is true, surely $\mathrm{BET}^{\vee}$ and $\mathrm{BET}^{\supset}$ should be true too! Since $\mathrm{BET}^{\vee}$ and $\mathrm{BET}^{\supset}$ are not true, BET should not turn out true either.

We claim that intuition conflates the deterministic but chaotic case, where present utterances of BET, $\mathrm{BET}^{\vee}$ and $\mathrm{BET}^{\supset}$ are all true, with the genuinely chancy indeterministic case, where present utterances of $\mathrm{BET}, \mathrm{BET}^{\vee}$, and $\mathrm{BET}^{\supset}$ are all indeterminate. It would not be surprising for our intuitions about chance to be mistaken in this way. Many of our everyday experiences with chance are with the deterministic, chaotic kind-roulette wheels, dice, and the like-so our intuitions

\footnotetext{
7 Phillips (2007) makes a similar point.
} 
are likelier to give true verdicts in these cases than in unusual, indeterministic ones. Even in deterministic cases, intuition does not have a stellar track record. We are often tempted by our gut to commit the Gambler's fallacy, where we believe a run of losses make a win on the next turn more likely. Our susceptibility to various fallacies involving probabilities is a sign that we should treat our intuitions with caution when it comes to chance.

The reader might remain skeptical. ${ }^{8}$ The coin toss and the bet are supposed to be independent - they might happen very far apart, timed so that it is impossible for any signal to pass between them. Doesn't our proposal make them counterfactually dependent? In particular, doesn't it make both of the following counterfactuals come out true?

TAILS If I had bet tails, the coin would have landed heads.

HEADS If I had bet heads, the coin might not have landed heads.

A theory that endorsed both HEADS and TAILS would indeed be committed to a worrying counterfactual dependence between the coin toss and the bet. (HEADS and TAILS taken together don't say that betting differently would have influenced the outcome of the coin toss, but they say that betting differently might have influenced the outcome of the coin toss, and that's bad enough.) And our theory is committed to HEADS. But our theory is not committed to TAILS.

TAILS is a consequence of strong centering - the claim that when $A$ is true at $T$, then the only selected $A$ timeline at $T$ is $T$ itself. But according to our theory, strong centering is false. Even where the antecedent $A$ is true, our procedure for generating antecedent timelines requires us to prune all the parts of $T$ after the antecedent's truth value was settled, and consider all feasible ways of regrowing the shorter timeline that would have made $A$ true. $T$ will always be among the selected timelines generated in this way, but it won't always be unique. The most our Growing-Block theorist can assert is:

TAILS $^{-}$If I had bet tails, the coin might have landed heads.

But TAILS $^{-}$, taken together with HEADS, does not commit us to any kind of counterfactual dependency between the bet and the coin toss.

Our response to Morgenbesser cases is closely related to our response to Lewis's inflexibility worry. Both Lewis's intuitions about the possibility of time travel and the Morgenbesser intuition about BET rest on the thought that sometimes, when we consider the antecedent of a counterfactual, we can hold later events fixed, while allowing earlier events to vary. In terms of our celery metaphor, instead of cutting off the end of the 'celery stick' timeline to remove the dodgy bit where the antecedent is false, we imagine cutting dodgy bits out of the middle, while leaving both the earlier bit and the later bit intact. We side with the Growing-Block intuition that the past is immutable (hence our response to the inflexibility worry) while the future is mutable (hence our response to the Morgenbesser worry).

\footnotetext{
${ }^{8}$ We thank an anonymous referee for pressing the objection.
} 
If the reader is still unconvinced, our formal semantics can be modified to accommodate the Morgenbesser intuition, the Lewis intuition, or both. We can accommodate the Lewis intuition by replacing our selection function with one based on overall similarity of timelines-as Lewis himself suggests for worlds (Lewis 1979). Likewise, we can accommodate the Morgenbesser intuition by modifying steps 2 and 3 in our characterization of the selection function to yield a new procedure for evaluating a counterfactual of the form $A \square \longrightarrow C$.

1. Find a time $e_{A}$, located shortly before the beginning of the span [that $A$ is about].

$2 *$. Remove all the parts of the timeline after $e_{A}$-thus generating a pruned timeline that represents the concrete block the way it was before it grew large enough furnish a falsemaker (or truthmaker) for the antecedent. But keep a list of propositions $L$ which are true of the times after $e_{A}$, and which are to be held fixed-perhaps all those propositions causally independent of $A$.

$3^{*}$. Consider all the feasible extensions of this shorter timeline-all the ways the concrete block might have grown-in which $A$ is true and in which all the propositions in $L$ are true. The timelines generated by this method are our selected timelines.

4. If $C$ is true in all of the resulting timelines, then $A \square \longrightarrow C$ is true in the original timeline; if $C$ is false in all of the resulting timelines, then $A \square \longrightarrow C$ is false in the original timeline; otherwise, $A \square \longrightarrow C$ is neither true nor false.

Both maneuvers come at a cost, however. Our preferred characterization of the selection function secures a tight link between indicative and counterfactual conditionals. Lewis's modification breaks the link in cases where the most similar timelines to the actual timeline at which the antecedent is true have different pasts (and are therefore not feasible). Our Morgenbesser modification breaks the link in cases where the truth of the consequent is affected by an event whose occurrence was determined later than the antecedent, but by a different causal route.

So we are inclined to the simpler semantics, which gives the traditional answer about BET in deterministic cases, and a slightly surprising answer about BET indeterministic cases. All this talk of indeterminism, however, might lead the reader to wonder how we deal with the nomological modality of chance.

\subsection{Chance}

So far we have divided propositions into three categories: the true, the false, and the indeterminate. But there are distinctions among indeterminate propositions. Some, while not quite true, are overwhelmingly likely. To take a practical example, consider the disposal of nuclear waste. The IAEA safety guide on the classification of nuclear waste (IAEA 2009, 4-5) distinguishes six level categories. For low level waste, the IAEA requires 'robust isolation and containment for up to a few hundred years', followed by 'disposal in engineered near surface facilities'. For high level waste, the IAEA requires 'disposal in deep, stable geological formations usually 
several hundred metres or more below the surface' This is presumably because somebody with a fresh container of low level waste is justified in asserting

LLW This low level waste will decay within 200 years to a relatively safe level of activity concentration.

while somebody with a fresh container of long-lived nuclear waste is not justified in asserting

HLW This high level waste will decay within 200 years to a relatively safe level of activity concentration.

But assuming that radioactive decay is fundamentally indeterministic, and that these decisions take place at the present time, both LLW and HLW express propositions with indeterminate truth values. What, then, explains the difference between them? The answer seems to lie in their different objective chances. To make sense of this difference, the Growing-Block theorist will need an account of objective chance. ${ }^{9}$

They might borrow some basic insights from Lewis (1994), who draws a connection between chances and laws. We suggest that the laws include not just deterministic claims, but additional history-to-chance conditionals of the form "If $H$, then $C H$ ", where $H$ is a complete proposition about history up to a certain time, and $\mathrm{CH}$ is a proposition about chances at that time. (Lewis requires that the conditional be "strong" in its modal import; we can accomplish this by saying that it is a material conditional that is true as a matter of law.) And we place another consistency requirement on laws: where the laws entail a history-to-chance conditional $H \supset(\operatorname{ch}(\phi)>0)$, they do not entail $\neg(H \wedge \phi)$.

We will follow Lewis in relativizing chances to both worlds and times. The chances are contingent, hence relative to a world. (For example, suppose a physicist has trapped a positively charged ytterbium ion in an excited ${ }^{2} P_{1 / 2}^{o}$ state. With probability 0.995 , the ion will decay from the excited state back to the ground state; with probability 0.005 , it will get stuck in a 'metastable' state (Olmschenk et al. 2009). But suppose the ion had instead started in the metastable state. Then its probability of decaying to the ground state would have been 0 , and not 0.995 .) The chances are also changeable, hence relative to a time. (In the ion example, if the ion does get stuck in the metastable state, its chance of decaying back to the ground state will drop to from 0.995 to roughly 0).

The Growing-Block theorist can achieve both world-relativity and time-relativity by indexing chances to timelines (worlds with a built-in present). At a first pass, we can say the chance function $c h_{T}$ at a timeline $T$ assigns a number between 0 and 1 to

\footnotetext{
${ }^{9}$ Notice that not all chances are problematic for the Growing-Block theorist. Many deterministic systems exhibit stable probabilities that are good deservers for the name 'objective chance'-though see Schaffer (2007) for a contrary argument. It is only indeterministic chances that pose a problem (if such things exist). But suppose that our world is both indeterministic and chancy. What can the Growing-Block theorist say about indeterministic chance?
} 


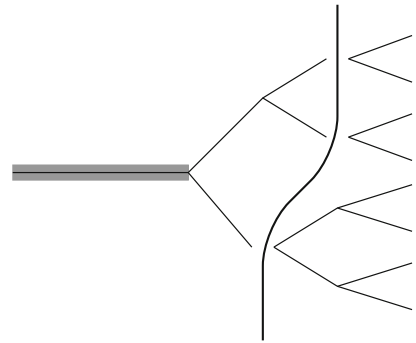

$\longleftarrow$ Earlier

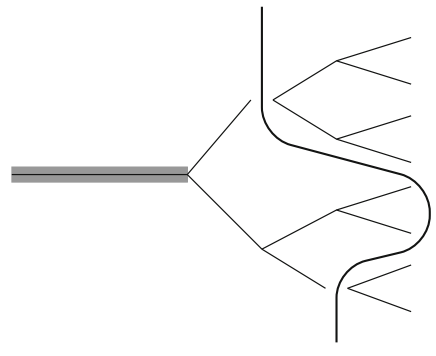

$\longleftarrow$ Earlier $\quad$ Later $\longrightarrow$

Fig. 3 Two ways of generating a partition

each of the timeline's feasible extensions. ${ }^{10}$ Where $T$ is a timeline and $T^{*}$ is a feasible extension of $T, c h_{T}\left(T^{*}\right)$ measures $T^{*}$ 's objective chance. The greater an ersatz timeline's chance at the actualized timeline, the more likely it is to come to represent the concrete block.

The Growing-Block Theorist needs the chances to be well-behaved; in particular, where some options form a partition (that is, an exhaustive set of mutually exclusive alternatives), their chances should add up to 1 . But what is a partition, understood things in terms of timelines? Unlike ordinary possible worlds, timelines are not mutually exclusive. Where one timeline is an extension of another, there is a sense in which the two distinct timelines are compatible: both may come to represent the same concrete block in different phases of its growth. So while the feasible extensions of a timeline exhaustively represent ways that the timeline could grow, they don't compose a set of mutually exclusive alternatives.

Where $T$ is a timeline, we can define a partition $\mathcal{A}$ on $T$ as any set of timelines such that

(i) For all $T^{*} \in \mathcal{A}, T^{*}$ is an extension of $T$,

(ii) For all $T^{*}, T^{\prime} \in \mathcal{A}, T^{*}$ is not an extension of $T^{\prime}$, and

(iii) For every $T^{*}$ that is an extension of $T$, there exists a $T^{\prime} \in \mathcal{A}$ such that either $T^{*}$ is an extension of $T^{\prime}$, or $T^{\prime}$ is an extension of $T^{*}$.

In intuitive pictorial terms, if you take the ersatz tree structure generated by $T$ and lop off some of its branches, the remaining limbs constitute a truncated tree. Any path that stretches all the way to the cut, or to a leaf that was not lopped off, represents an alternative timeline. Figure 3 depicts two ways of generating a partition on a tree; in each image, the partition is the set of all timelines that extend from the root of the tree to the wavy line.

\footnotetext{
${ }^{10}$ We will need to make two discreteness assumptions, in order to ensure that our first pass works. First, we assume that time is discrete: between any two times on a timeline, the number of intermediate times is finite. Second, we assume that the possibilities are discrete: no timeline has more than finitely many extensions which are longer than it by only one ersatz time. We believe that our account could be generalized using measure theory, but we want to get the simple case right first.
} 
Given this definition of a partition, we require that chance functions satisfy the following constraints.

1. For all timelines $T$ and $T^{*}, c h_{T}\left(T^{*}\right) \geq 0$.

(No timeline assigns another timeline chance less than 0 ).

2. Where $\left\{T_{1}, T_{2}, \ldots T_{n}\right\}$ is a partition on $T, \sum_{i=1}^{n} c h_{T}\left(T_{i}\right)=1$.

(The chances of timelines in a finite partition always sum to 1 ).

3. If $T_{2}$ is an extension of $T_{1}$ and $T_{1}$ is an extension of $T_{0}$, then $c h_{T_{0}}\left(T_{2}\right)=c h_{T_{0}}\left(T_{1}\right) \times c h_{T_{1}}\left(T_{2}\right)$. (The chances of timelines obey a version the multiplicative formula: the $T_{0}$ chance of getting to $T_{2}$ is the $T_{0}$ chance of getting as far as $T_{1}$, multiplied by the $T_{1}$ chance of getting to $T_{2}$.)

Given an assignment of chances to timelines, we can extend the chance function to assign chances to propositions - or at least some of them. We will assume that chances attach to tenseless propositions. (So instead of saying that the proposition that it is nighttime in Kent cycles between chance 0 and chance 1 as the block grows, we will say that the proposition that it is nighttime in Kent at midnight GMT on August 25th, 2020 has chance 1, now and hereafter.) And we assume that chances attach to propositions with the heredity property-propositions that, as soon as they become true at a time in a timeline, remain true at that time in all extensions of that timeline. (So the proposition that Sky Masterson rolls six on a particular toss of the die has a chance, but the proposition that Sky Masterson is in the objective present has no chance-not even chance 0.)

For any suitable proposition $A$ at any timeline $T$, the chance of $A$ relative to a partition $\mathcal{A}$ on $T$ is the sum of the chances of timelines at which $A$ is true. In other words,

$$
\operatorname{ch}_{\mathcal{A}} A=\sum_{T \in \mathcal{A}} \operatorname{ch}(T) \times v_{T}(A)
$$

Say that $\mathcal{A}$ settles $A$ just in case for every $T \in \mathcal{A}$, either $A$ is true at $T$ or $\neg A$ is true at $T$. Then the chance of a proposition is equal to its chance at an arbitrary partition that settles it. Given constraints 1 and 3 on the chance function, it does not matter which partition we choose. ${ }^{11}$

\footnotetext{
11 Sketch of a proof: Consider two partitions $\mathcal{A}_{1}$ and $\mathcal{A}_{2}$ on $T$, both of which settle $A$. We can show that the chance of $A$ is the same whether calculated using $\mathcal{A}_{1}$ or $\mathcal{A}_{2}$.

$\mathcal{A}_{1}$ and $\mathcal{A}_{2}$ will have a meet $\mathcal{A}_{\wedge}=\left\{T^{*} \in \mathcal{A}_{1} \cup \mathcal{A}_{2}: T^{*}\right.$ is not an extension of any $\left.T^{\prime} \neq T^{*} \in \mathcal{A}_{1} \cup \mathcal{A}_{2}\right\}$.

We can show that $\mathcal{A}_{\wedge}$ is a partition that settles $A$. $\mathcal{A}_{\wedge}$ satisfies each clause in the definition of a partition.

(i) $\mathcal{A}_{\wedge} \subseteq \mathcal{A}_{1} \cup \mathcal{A}_{2}$. Since $\mathcal{A}_{1}$ and $\mathcal{A}_{2}$ satisfy (i), so does $\mathcal{A}_{\wedge}$.

(ii) Guaranteed by the definition of $\mathcal{A}_{\wedge}$.

(iii) Suppose $T^{*}$ is an extension of $T$. Then either $T \in \mathcal{A}_{1}$, or $T \in \mathcal{A}_{2}$. Since $\mathcal{A}_{1}$ and $\mathcal{A}_{2}$ satisfy (iii), $T$ must either be an extension of some timeline in $\mathcal{A}_{1}$, an extension of some timeline in $\mathcal{A}_{2}$, or a timeline with extensions in both $\mathcal{A}_{1}$ and $\mathcal{A}_{2}$. In the first two cases, $T$ is an extension of some timeline in $\mathcal{A}_{\wedge}$ (since every timeline in $\mathcal{A}_{1} \cup A_{2}$ is an extension of $\mathcal{A}_{\wedge}$, and extension is transitive). In the third case, $T$ has an extension in $\mathcal{A}_{\wedge}$ (namely itself). Thus, for any arbitrarily chosen $T \in \mathcal{A}_{\wedge}$, $\mathcal{A}_{\wedge}$ contains either a timeline that extends $T^{*}$ or that is extended by $T^{*}$.
}

Furthermore, $\mathcal{A}_{\wedge}$ settles $A$, since every timeline in $\mathcal{A}_{\wedge}$ is either in $\mathcal{A}_{1}$ or in $\mathcal{A}_{2}$, both of which settle $A$. 
Constraints 1-3 guarantee that the chances of propositions satisfy the Kolmogorov axioms ${ }^{12}$ :

Non-Negativity For all propositions $A$ such that $c h_{T}(A)$ is defined, $c h_{T}(A) \geq 0$. (Every proposition has a chance greater than or equal to 0 .)

Tautology If $(A)$ is a necessary truth, then $\operatorname{ch}_{T}(A)=1$.

(All necessary truths have chance 1.)

Footnote 11 continued

We can then create a series of partitions from $\mathcal{A}_{1}$ to $\mathcal{A}_{\wedge}$ each of which gives the same chance for $A$ at $T$. Number the timelines in $\mathcal{A}_{\wedge}-\mathcal{A}_{1}$ as $T_{1}, T_{2}, \ldots T_{n}$, and let us define recursively:

$$
\begin{aligned}
\mathcal{B}_{i} & =\left\{T^{*} \in \mathcal{A}_{i}: T^{*} \text { isanextensionof } T_{i}\right\} \\
\mathcal{A}_{i+1} & =\left(\mathcal{A}_{i} \cup T_{i}\right)-\mathcal{B}_{i}
\end{aligned}
$$

We know that $\mathcal{B}_{i}$ is a partition on $T_{i}$. Therefore by constraint 2 on chances,

$$
\sum_{T^{*} \in \mathcal{B}_{i}} c h_{T_{i}}\left(T^{*}\right)=1
$$

By constraint 3 on chances,

$$
\sum_{T^{*} \in \mathcal{B}_{i}} c h_{T}\left(T^{*}\right)=\operatorname{ch}_{T}\left(T_{i}\right)
$$

By the definition of chance for propositions, $c h_{T}(A)$ as calculated using $\mathcal{A}_{i}$ is

$$
\sum_{T^{*} \notin B_{i}} c h_{T}\left(T^{*}\right) \times v_{T^{*}}(A)+\sum_{T^{*} \in B_{i}} c h_{T}\left(T^{*}\right) \times v_{T^{*}}(A)
$$

while $\operatorname{ch}_{T}(A)$ as calculated using $\mathcal{A}_{i+1}$ is

$$
\sum_{T^{*} \notin B_{i}} c h_{T}\left(T^{*}\right) \times v_{T^{*}}(A)+c h_{T}\left(T_{i}\right) \times v_{T}(A)
$$

Since $\mathcal{A}_{\wedge}$ settles $A, A$ is either true in $T_{i}$ (and by heredity, every timeline in $\mathcal{B}_{i}$ ) or false in $T_{i}$ (and by heredity, every timeline in $\mathcal{B}_{i}$ ). In either case,

$$
\sum_{T^{*} \in B_{i}} c h_{T}\left(T^{*}\right) \times v_{T^{*}}(A)=c h_{T}\left(T_{i}\right) \times v_{T}(A)
$$

so that $\operatorname{ch}_{T}(A)$ is the same whether calculated using $\mathcal{A}_{i}$ or $\mathcal{A}_{i+1}$, for arbitrary $i$. Thus $c h_{T}(A)$ is the same whether calculated using $\mathcal{A}_{1}$ or $\mathcal{A}_{\vee}$. By parity of reasoning it is the same whether calculated using $\mathcal{A}_{2}$ or $\mathcal{A}_{\vee}$, and by transitivity of identity, it is the same whether calculated using $\mathcal{A}_{1}$ or $\mathcal{A}_{2}$.

${ }^{12}$ Non-Negativity follows from constraint 1 , and the fact that a sum of non-negative numbers is always a non-negative number.

Tautology follows from 2, together with the observation that a necessary truth is true in every timeline in every partition.

Finite Additivity follows from 2, together with the assumption that every proposition will be settled in a finite amount of time. The chance of $A \vee B$ is the sum of the chances of the timelines at which $A \vee B$ is true in any partition that settles $A \vee B$. Given our finiteness assumption, some such partition will settle $A$ and settle $B$. Every timeline where $A \vee B$ is true will be one where either $A$ is true or be is true. Where $A$ and $B$ are incompatible propositions, no timeline in the partition will be one where both $A$ is true and $B$ is true. So within the partition, the timelines where $A \vee B$ is can be divided without remainder into those where $A$ is true and those where $B$ is true. Now assumption 2 guarantees that the chance of $A \vee B$ is the sum of the chance of $A$ and the chance of $B$. 
Finite Additivity Where $A$ and $B$ are incompatible propositions, $c h_{T}(A \vee B)=$ $c h_{T}(A)+c h_{T}(B)$. (The chance of a disjunction of two exclusive alternatives equals the sum of their chances.)

How does all of this help with the LLW and HLW examples that motivated our discussion of chance? We can treat the chances of propositions as generalized truth values. This enables us to distinguish among indeterminate propositions in a more fine-grained way, according to their degrees of truth.

At any given time, each tensed proposition can be associated with a tenseless one. (For example, on January 1st 2001, the tensed proposition there will be a sea battle tomorrow corresponds to the tenseless proposition there is a sea battle on January 2nd 2001.) We can say that the truth value of a tensed proposition at a time equals the chance of the corresponding tenseless proposition. Since LLW is associated with a high-probability tenseless proposition, it is close to true; since HLW is associated with a low-probability tenseless proposition, it is close to false. Assuming that what is guaranteed by the past and the laws has chance 1, our supervaluationist theory of truth falls out as a special case.

A link between chance and truth would explain the connection between chance and partial belief. Someone omniscient-who knows all the basic facts there are to know-should believe propositions to the extent that they are true. Likewise, someone who is omniscient by the lights of the Growing-Block theory-who knows everything about the past, the present, and the laws—-should believe propositions to the extent that their chance is high. Thus, identifying chance with degree of truth explains the claims of Hájek (ms) and Pettigrew (2012) that while full belief aims toward truth, partial belief aims toward matching the chances. Furthermore, someone who is ignorant about a proposition should believe it to the extent that its subjectively expected chance is (i.e., expected truth value) is high, as the Principal Principle requires (see Lewis 1980). Thus, identifying chances to degrees of truth provides a natural explanation of their epistemic role.

\subsubsection{Comparison with Storrs McCall's view}

Our view is similar to Storrs McCall's view of probability in a branching time framework (McCall 1994). And indeed, the reader who prefers McCall's view of chance might substitute it for ours while leaving the rest of our theory intact. But we will argue that our view has a number of advantages.

McCall holds that the chance of a proposition $A$ at a time $e$ is equal to the ratio between the number of branches passing through $e$ on which $A$ is true, and the total number of branches passing through $e$. Our theory, like McCall's, can be seen as assigning weights to sets of branches in a branching model structure. However, we hold that the chance of a set of branches is not a ratio, but a measure.

McCall's ratio theory is vulnerable to a number of objections, which the measure theory gives new ways of circumventing. First, it seems as though there could be irrational chances (such as the chance that a dart hits a circle inscribed in a square dart board, where its probability of hitting each part of the dartboard is proportional 
to that part's area. But irrational numbers by definition cannot be expressed as ratios.

McCall has a proposal for accommodating irrational probabilities. We can imagine trials like the dart trial (or the quantum mechanical trials that McCall envisions) as infinite 'decenary' trees, which branch into ten at each uncertain juncture. Consider an arbitrary real number $r$ in decimal expansion; we can design an infinite tree that assigns probability $r$ to some proposition $A$. Where $r_{1}$ is the first digit of $r$, that the first time the tree branches, $r_{1}$ of the branches decide that $A$ is true, $9-r_{1}$ of the branches decide that $A$ is false, and on the remaining branch, $A$ is undecided. Where $r_{2}$ is the second digit in $r$, the undecided branch divides into $r_{2}$ branches that make $A$ true, $9-r_{2}$ branches that make $A$ false, and so on.

This model gets the right formal results, but at a cost. Many of the events that McCall is attempting to model (quantum measurements with two possible outcomes) appear to be single-shot trials rather than sequential trials-it is not as though a given quantum measurement has separable beginning, middle, and end phases. But McCall's decenary trees represent them as infinite sequences of trials. This may be a harmless artifact of the representation rather than a serious hazardbut it's an obstacle that we can avoid altogether by viewing chance as a measure.

A second problem for McCall is that there may be too many outcomes for the ratio theory to make sense. Consider again a dart with a point-sized tip, about to be thrown at a dart board. Since there are infinitely many points on the dart board, it is dubious whether the concept of a ratio makes sense. The set of points on the left hand of the dart board is equal in number to the set of points on the whole dart board, but this does not (presumably) entail that the chance that the dart lands on the left-hand side of the board is equal to the chance that the dart hits the board at all. Moreover, McCall's method will not help, since the number of points on the dart board is uncountable. Our current framework is not equipped to deal with such infinite cases either, but it is easier to see how our theory might be generalized; after all, measures can be generalized to infinite spaces while counts cannot.

A third problem is that McCall's theory presents particular difficulties when combined with our view of ersatz timelines. Imagine a timeline in which a genuinely chancy coin is about to be tossed, and will land either heads or tails. Suppose that neither outcome subdivides into more than one feasible possibility. Does it follow that the coin is unbiased? It does not seem to-even if the coin were biased, there would still be two possible outcomes, heads and tails, with different probabilities. But we run into trouble when we combine McCall's theory with the view of that timelines are ersatz possible worlds. We could posit multiple indiscernible concrete timelines to make the numbers come out right, but there are no distinct but discernible ersatz timelines. Adding McCall's ratio theory to the our view of ersatz timelines yields the result that the chance of heads must be $1 / 2$, since exactly one of the two possible outcomes is a heads outcome.

While our view boasts advantages over McCall's view, both theories of chance have the power to significantly strengthen our overall Growing-Block theory of the nomological package. Where before our model flattened the distinction between the overwhelmingly likely and the overwhelmingly unlikely - treating both as equally 
indeterminate - we can now assign chances and credences to future possibilities, or counterfactual ones.

\subsection{Dispositions and causation}

There are two key elements of the nomological package we have not yet addressed: dispositions and causation. Each of these might fit into one of two places in our picture: either at the bottom of our explanatory hierarchy, as a lawmaker; or at the top, as something with a counterfactual analysis. There is also a third possible place for causation: it might be tightly coupled with feasibility in a way that enables it to explain the growth of the block. The placement of causation and dispositions in the hierarchy is independent. Let us begin with dispositions.

\subsubsection{Dispositions}

We often appeal to dispositions in order to explain events. We might explain why the cricket ball broke the window by appealing to a feature of windows - that they are breakable — or a feature of cricket balls - that they are disposed to break fragile things when moving at speed. This is very useful. Not only does it help us explain past events, like the breakings of windows, but it helps us plan for the future: when we play cricket near windows, perhaps a tennis ball would be better than a wooden ball, or, when we install windows near the cricket field, we should also install protective netting.

How are dispositions connected to the rest of the nomological package? We see two options. First, dispositions might be susceptible to a reductive analysis in terms of counterfactuals (see Choi 2008). This analysis may be holistic: even if the dispositions of the cricket ball cannot be analyzed in terms of counterfactuals about the cricket ball, the counterfactual truths about the universe might determine the dispositions of everything in the universe. Alternatively, dispositions themselves could be lawmakers, and could ground facts about laws, counterfactuals and other elements of the nomological package.

Both possibilities - that dispositions ground laws (and thereby feasibility and counterfactuals), and that counterfactuals ground dispositions (and are in turn grounded by laws and feasibility)_posit a tight modal connection between counterfactuals and dispositions. This renders our proposal susceptible to an objection from (Mumford and Anjum, ms, 175), who deny the existence of any such connection. They argue that the dispositional modality is sui generis and irreducible. We can reconstruct two arguments from their manuscript: one for the conclusion that counterfactuals cannot ground dispositions, and one for the conclusion that dispositions cannot ground laws.

Consider first the case that counterfactuals cannot ground dispositions. Mumford and Anjum reject a reductive analysis of dispositions in terms of counterfactuals because, they claim, dispositions always allow a chance that something else will interfere.

On one way of understanding this claim, we sympathise. If I tell you that the window is fragile and the cricket ball is hard, I have not said enough to establish that the window would shatter if struck by the cricket ball. Perhaps if the window were 
struck by the cricket ball, some outside intervention would prevent the breaking: the cricket ball would be deflected by a passing bird, or fly in at a fortuitous angle and strike one especially strong point in the glass, or an agile custodian would step in and reinforce the window with just the right protective backing, or ... At best, the connection between the disposition claim and the corresponding counterfactual holds only ceteris paribus.

But the claim that facts about dispositions are grounded by facts about counterfactuals is not the claim that facts about dispositions are grounded only by the facts about some restricted set of counterfactuals. The dispositions of the cricket ball and the window need not be grounded only in counterfactuals about the window and the ball. Rather, the facts about dispositions could be grounded by all the facts about counterfactuals: the window's fragility may be grounded partly in the fact that if the ball were to strike the window and a passing bird were to deflect it only slightly, then the window would still break.

Mumford and Anjum might push back: some dispositions are stronger than others: a sledgehammer can have a stronger disposition to crack a walnut than a shoe, even if both succeed in cracking it. But how could there be a counterfactual analysis of dispositions' comparative strength? We propose that comparative strength can be explained in terms of counterfactual robustness; there are more counterfactually close possibilities where we succeed in cracking the walnut with a sledgehammer than counterfactually close possibilities where we succeed in cracking it with a shoe. We need not claim that humans will be in a position to know all the truths about counterfactuals, even in principle, as long as whatever grounds the facts about the counterfactuals also grounds the facts about dispositions.

Now consider the argument against our second option-that dispositions are lawmakers. Mumford and Anjum claim that dispositions cannot necessitate: the fragility of the window and the hardness of the cricket ball cannot ground a necessary connection between the cricket ball's striking the window and the window's breaking. There is always a possibility that something could interfere between the striking and the breaking. Although the dispositions of the cricket ball and the window may ground something, they cannot ground anything with the strength of a law-i.e., a necessary universal generalization.

We agree that the dispositions of the window and the cricket ball alone are not enough to establish a necessary connection between the cricket ball's striking the window and the window's breaking. But this is not enough to show that the dispositions (in general) are unfit to ground the laws (in general). Again, if there is grounding here, it is likely to be global: the facts about all dispositions of past and present things (including totality facts) are enough to ground necessary connections between past and present events and future ones. Taken together, the dispositional facts about the past and present do necessitatethey rule out some future courses of events as infeasible.

\subsubsection{Causation}

We will consider one last element in the nomological package: causation. Hall (2004) identifies two concepts of causation in the philosophical literature, which seem to fit into different places in our grounding hierarchy. 
One concept of causation is counterfactual (see Menzies 2014 for an overview). Counterfactual theories of causation have been invoked to explain the temporal asymmetry of causation, our ability to manipulate effects through their causes, and the nature of causal explanation. Such theories would place counterfactuals at the top of our grounding hierarchy, to be analyzed in counterfactual terms.

The other concept of causation, captured by process theories, characterizes causation as a sort of production or necessitation that cannot be captured by counterfactuals alone. According to these theories, causation consists in the transfer of a mark (Salmon 1984) or quantity (Skyrms 1980; Dowe 1992; Salmon 1994) across a spatiotemporally connected region. Process theories distinguish between genuine causation on the one hand (which requires a continuous line along which causes and effects interact); and omission (e.g., a condemned building remaining intact because the demolition team didn't show up), prevention (e.g., a building's being condemned because the owner failed to perform necessary maintenance and refurbishment) and pseudo-causation (e.g., the shadow of a wall appearing to crumble when 'hit' by the shadow of a wrecking ball) on the other.

One place to locate process causation in our hierarchy is above the laws, and below feasibility: causal connections are constrained by the laws, and in turn constrain what is feasible. This would allow us to explain causal processes by appeal to the laws. It would also enable causation to fulfill the role envisioned by Schaffer (2004) of underpinning the selection function used to generate verdicts about counterfactual dependence. ${ }^{13}$

But there is another way to combine process theories with the Growing-Block theory: we might claim that causation is what enables the block to grow. The laws in our model constrain how time passes, provided it does (only those possibilities which are feasible can be actualized), but don't we need an additional ingredient to guarantee that time will pass? Some Growing-Block theorists, like Broad (1923), deny that any such additional ingredient is needed, claiming that the growth of the block is primitive. Others, like Mackie (1980) hold that what we need is causation, which turns the potentiality of the future into the actuality of the past by bringing more of the concrete Growing-Block into existence.

On this third interpretation, causation is intimately connected with feasibility, explaining why the block grows in exactly the way it does. One could understand this as causation (together with the laws) grounding feasibility-the feasible timelines are just the ones that correctly describe forms that causation might compel the block to take in accordance with the laws. Alternatively, one could understand this as feasibility grounding causation-causation is what will inevitably push the block from something that actualizes the current timeline to something that actualizes another feasible timeline. Since either description seems apt, we have drawn this possible relationship between causation and feasibility as an undirected edge in Fig. 2 (Sect. 2).

\footnotetext{
13 Schaffer suggest that we embrace circularity, and also give a counterfactual analysis of causation. But as he notes, this circularity is not mandatory.
} 
There are three places, then, where causation might fit into the Growing-Block theory: it might be grounded by relations of counterfactual dependence; it might be grounded by the laws and ground feasibility (and thereby ground counterfactual dependence); or it might be intimately connected with feasibility in a way that explains the growth of the block itself. Perhaps there are even multiple entities called 'causation', with each fitting into a different place. We do not pretend to have a complete analysis, but we have cleared a space (or three) for causation in a nonHumean Growing-Block theory.

\subsection{Odds and ends}

We have unpacked all the items that philosophers typically bundle into the nomological package, but there might be more rattling around inside. The nomological package is not merely a heterogenous list of categories, but a set of categories that share a certain modal character. Might there be other categories with this modal character too? We propose a few interesting candidates for further investigation.

For the Growing-Block theorist, persistence might be modal in the relevant sense. Mackie (1980, 221) says that "basic laws of working are, in part, forms of persistence"; persistence is necessary to explain the continued operation of underlying processes, and the continued existence of objects.

Structural equations, a recent arrival on the philosophical scene, also seem qualified to be part of the nomological package. These seem to have the same dual character as dispositions and causation; some authors place them at the top of our grounding hierarchy, assuming that they are shorthand for sets of counterfactuals (Hitchcock 2001); while others place them near the bottom of our grounding hierarchy, and try to analyze counterfactuals in terms of them (Hiddleston 2005; Pearl 2009).

We aim to break new ground in this paper, and that inevitably leaves uncertainty about the shape of the landscape. This paper is therefore, in part, an invitation to join us in exploring. We have drawn attention to persistence and structural equations because they are neglected parts of the nomological package that deserve more attention. We hope that readers will take up our invitation to investigate them further.

\section{Conclusion}

We have shown that five important elements of the nomological package-laws, counterfactuals, chances, dispositions, and counterfactuals-needn't be a problem for the Growing-Block view. Taking laws as primitive, we have shown that the Growing-Block view has the resources to provide an account of possibility, and a natural semantics for non-backtracking causal counterfactuals. We have shown how objective chances might ground a more fine-grained concept of feasibility, and furnished a few places in the structure where causation and dispositions might fit.

There are a few outstanding problems for our account, which deserve further research. The account doesn't yet provide a fully general semantics for counterfactuals. It's somewhat open-ended what should be included in the nomological package, and we haven't yet pinpointed where causation and dispositions belong. 
Nonetheless, we have made considerable headway. The Growing-Block view provides the resources to explain the close link between modality and tense, so that it predicts modal change as time passes. It brings various elements of the nomological package together. It provides modeling tools that the Growing-Block theorist can use to capture truths about laws, chances, and counterfactuals in a unified formal framework-including the link between indicatives and counterfactuals, and the correct verdicts about Morgenbesser cases. It allows us to see where causation and dispositions might fit in. And this same account lets us capture not only what the future might hold for us, and also what might have been. By doing all this, the Growing-Block view becomes a more appealing philosophical theory-one with a coherent story to tell.

Open Access This article is distributed under the terms of the Creative Commons Attribution 4.0 International License (http://creativecommons.org/licenses/by/4.0/), which permits unrestricted use, distribution, and reproduction in any medium, provided you give appropriate credit to the original author(s) and the source, provide a link to the Creative Commons license, and indicate if changes were made.

\section{References}

Adams, E. W. (1970). Subjunctive and indicative conditionals. Foundations of Language, 6, 89-94.

Adams, R. M. (1974). Theories of actuality. Noûs, 8(3), 211-231.

Briggs, R. A. (2012). Interventionist counterfactuals. Philosophical Studies, 160(1), 139-166.

Briggs, R. A., \& Forbes, G. A. (2012). The real truth about the unreal future. Oxford studies in metaphysics. Oxford: Oxford University Press.

Briggs, R. A., \& Forbes, G. A. (2017). The growing block: Just one thing after another? Philosophical Studies, 174(4), 927-943.

Broad, C. D. (1923). Scientific thought. London: Routledge and Kegan Paul.

Brogaard, B., \& Salerno, J. (2013). Remarks on counterpossibles. Synthese, 190(4), 639-660.

Button, T. (2006). There's no time like the present. Analysis, 66(290), 130-135.

Cartwright, N. (1999). The dappled world: A story of the boundaries of science. Cambridge: Cambridge University Press.

Chakravartty, A. (2003). The dappled world: A study of the boundaries of science. Philosophy and Phenomenological Research, 66(1), 244-247.

Choi, S. (2008). Dispositional properties and counterfactual conditionals. Mind, 117(468), 795-841.

Correia, F., \& Rosenkranz, S. (2013). Living on the brink, or welcome back, growing block! Oxford studies in metaphysics (Vol. 8, p. 333). Oxford: Oxford University Press.

Dorothy, E. (2004). Counterfactuals and the benefit of hindsight. In P. Dowe \& P. Noordhof (Eds.), Cause and chance: Causation in an indeterministic world. London: Routledge.

Dowe, P. (1992). Wesley salmon's process theory of causality and the conserved quantity theory. Philosophy of Science, 59, 195-216.

Forbes, G. A. (2016). The growing block's past problems. Philosophical Studies, 173(3), 699-709.

Hájek, A. (2011). A puzzle about partial belief. MS. http://fitelson.org/coherence/hajek_puzzle.pdf

Hall, N. (2004). Two concepts of causation. In J. Collins, N. Hall, \& L. Paul (Eds.), Causation and counterfactuals (pp. 225-276). Cambridge: MIT Press.

Hiddleston, E. (2005). A causal theory of counterfactuals. Noûs, 39(4), 232-257.

Hitchcock, C. (2001). The intransitivity of causation revealed in equations and graphs. The Journal of Philosophy, 98(6), 273-299.

IAEA. Classification of radioactive waste. General Safety Guide GSG-1, IAEA, December 2009. http:// www-pub.iaea.org/MTCD/publications/PDF/Pub1419_web.pdf.. Accessed 21 Dec 2017.

Krakauer, B. (2012) Counterpossibles. PhD thesis, University of Massachusetts.

Leitgeb, H. (2011a). A probabilistic semantics of counterfactuals, part A. The Review of Symbolic Logic, 5(1), 26-84. 
Leitgeb, H. (2011b). A probabilistic semantics of counterfactuals, part B. The Review of Symbolic Logic, $5(1), 85-121$.

Lewis, D. K. (1973). Counterfactuals. Cambridge: Harvard University Press.

Lewis, D. K. (1979). Counterfactual dependence and time's arrow. Nô̂s, 13(4), 455-476. (Reprinted in Lewis 1986 with postscript, pp. 32-52) .

Lewis, D. (1980). A subjectivist's guide to objective chance. In R. Jeffrey (Ed.), Studies in inductive logic and probability (Vol. II). Berkeley: University of California Press. (Reprinted in Lewis 1986, pp. 83-113) .

Lewis, D. (1994). Humean supervenience debugged. Mind, 103(412), 473-490.

Loewer, B. (2007). Counterfactuals and the second law. In H. Price \& R. Corry (Eds.), Causation, physics and the constitution of reality: Russell's republic revisited (pp. 293-326). Oxford: Oxford University Press.

Mackie, J. L. (1980). The cement of the universe: A study of causation. Oxford: Clarendon Press.

McCall, S. (1994). A Model of the Universe. Oxford: Clarendon Press.

Menzies, P. (2014) Counterfactual theories of causation. In: EN Zalta (ed) The Stanford encyclopedia of philosophy, Spring 2014 edition. Metaphysics Research Lab, Stanford University. https://plato. stanford.edu/archives/spr2014/entries/causation-counterfactual/.

Morton, A. (2004). Indicative vs. subjunctive in future conditionals. Analysis, 64(4), 289-293.

Mumford, S. (2000). The dappled world: A study of the boundaries of science. Philosophy, 75(4), 613-626.

Mumford, S., \& Anjum, R. L. What tends to be: Essays on the dispositional modality. MS.

Nolan, D. (1997). Impossible worlds: A modest approach. Notre Dame Journal of Formal Logic, 38(4), 535-572.

Olmschenk, S., Hayes, D., Matuskevich, D. N., Mauns, P., Hoehring, D. L, Younge, K. C, et al. (2009). Measurement of the lifetime of the $6 p^{2} p_{1 / 2}^{o}$ level of $\mathrm{yb}^{+}$. Physical Review A, 80, 022502.

Pearl, J. (2009). Causality: Models inference, and reasoning (2nd ed.). Cambridge: Cambridge University Press.

Pettigrew, R. (2012). Accuracy, chance, and the principal principle. Philosophical Review, 121(2), 241-275.

Phillips, I. (2007). Morgenbesser cases and closet determinism. Analysis, 7(1), 42-49.

Salmon, W. (1984). Scientific explanation and the causal structure of the world. Princeton: Princeton University Press.

Salmon, W. (1994). Causation without counterfactuals. Philosophy of Science, 61(312), 297.

Schaffer, J. (2004). Counterfactuals, causal independence and conceptual circularity. Analysis, 64(4), 299-308.

Schaffer, J. (2007). Deterministic chance. British Journal for the Philosophy of Science, 58, 113-140.

Schulz, K. (2011). 'If you'd wiggled A, then B would've changed'. Synthese, 179(2), 239-251.

Skyrms, B. (1980). Causal necessity. New Haven: Yale University Press.

Slote, M. (1978). Time in counterfactuals. Philosophical Review, 87(1), 3-27.

Stalnaker, R. (1968). A theory of conditionals. In N. Rescher (Ed.), Studies in logical theory, Volume 2 of American philosophical quarterly monograph series. Oxford: Blackwell.

Stalnaker, R. (1981). A theory of conditionals. In W. L. Harper, R. Stalnaker, \& G. Pearce (Eds.), Ifs, volume 15 of The University of Western Ontario series in philosophy of science (Vol. 15, pp. 41-55). Dordrecht: D. Reidel.

Stalnaker, R. C., \& Thomason, R. H. (1970). A semantic analysis of conditional logic. Theoria, 36(1), 23-42.

Teller, P. (2002). Critical study: Nancy Cartwright's the dappled world: A study of the boundaries of science. Noûs, 36(4), 699-725.

Thomason, R., \& Gupta, A. (1981). A theory of conditionals in the context of branching time. In W. L. Harper, R. Stalnaker, \& G. Pearce (Eds.), Ifs, Volume 15 of The University of Western Ontario series in philosophy of science (pp. 299-322). Dordrecht: D. Reidel.

Tooley, M. (1997). Time, tense, and causation. Oxford: Oxford University Press.

Williamson, T. (2007). The philosophy of philosophy. Oxford: Blackwell Publishing.

Winsberg, E., Frisch, M., Darling, K. M., \& Fine, K. (2000). The dappled world: A study of the boundaries of science. Journal of Philosophy, 97(7), 403-408. 